\title{
Ecología poblacional de Crocodylus acutus en los sistemas estuarinos de San Blas, Nayarit, México
}

\section{Population ecology of Crocodylus acutus in the estuarine systems of San Blas, Nayarit, Mexico}

\author{
Helios Hernández-Hurtado ${ }^{1,3 *}$, José de Jesús Romero-Villaruel² y Pablo Simitrius Hernández-Hurtado ${ }^{1}$ \\ ${ }^{1}$ Universidad de Guadalajara, Centro Universitario de la Costa. Av. Universidad 203, Ixtapa. 48280 Puerto Vallarta, Jalisco, México. \\ ${ }^{2}$ Delegación Secretaría de Medio Ambiente y Recursos Naturales, Nayarit. Av. Allende 110, $2^{\circ}$ piso, Col. Oriente, 63000 Tepic, Nayarit, México. \\ ${ }^{3}$ Posgrado en Ciencias Biológicas y Agropecuarias, Universidad Autónoma de Nayarit. Ciudad de la Cultura, Amado Nervo, 63155, Tepic, Nayarit, \\ México. \\ *Correspondencia: helios@cuc.udg.mxyhhh0474@hotmail.com
}

\begin{abstract}
Resumen. El presente estudio se realizó en los meses de junio 2005 y febrero 2006 (estiaje), y en octubre 2005 y octubre 2007 (lluvias), en los esteros de San Blas. El objetivo fue caracterizar la población de cocodrilos a partir de su distribución y abundancia, El método utilizado consistió en recorridos nocturnos en los esteros, para contabilizar el número de individuos por kilómetro. Se realizaron 6 transectos recorriendo un total de 89 kilómetros. Los transectos A, B y C presentaron una densidad que osciló entre 2.68 y 4.31 ind $/ \mathrm{km}$ o 0.05 ind $/$ ha con 26 nidos activos, 6 tipos de vegetación y salinidad de $11.03 \%$ en estiaje a $4.92 \%$ en lluvias. Los transectos D, E y F presentaron una densidad que osciló entre 0.014 y $0.36 \mathrm{ind} / \mathrm{km}$ o 0.002 ind/ha, sin nidos, 1 tipo de vegetación y salinidad qosciló de 35.85\%o en estiaje a $24.56 \%$ en lluvias. Se obtuvo la similitud estadística en A, B y C=80\%, D y E= 70\% y en F=10\% respecto a los demás. La población estimada fue de 333 cocodrilos, registrando todas las clases-talla, donde el hábitat disponible presenta las características necesarias para que Crocodylus acutus realice su ciclo biológico.
\end{abstract}

Palabras clave: distribución, abundancia, cocodrilo de río, cocodrilo americano.

\begin{abstract}
This study was conducted in San Blas estuaries during June 2005 and February 2006 (dry season) and October 2005 and October 2007 (rainy season). The purpose was to describe the population of crocodiles according to its distribution and abundance. Spotlight surveys counting animals found per kilometer were done navigating 89 kilometers in 6 transects. Transects A, B and C had a density from 2.68 to 4.31 crocodiles/kilometer or 0.05 crocodiles/ ha, with 26 active nests, 6 different types of vegetation and salinity from $11.03 \%$ in dry season to $4.92 \%$ in rainy season. Transects D, E and F had a density from 0.014 to 0.36 crocodiles $/ \mathrm{km}$ or 0.002 crocodiles $/$ ha, any nest was found, 1 single type of vegetation and salinity from $35.85 \%$ in dry season to $24.56 \%$ in rainy season. Statistical similarity in transects $\mathrm{A}, \mathrm{B}$ and $\mathrm{C}$ was $80 \%$, in transects $\mathrm{D}$ and $\mathrm{E}$ was $70 \%$ and in transect $\mathrm{F}$ was $10 \%$. Crocodile population was estimated in 333 crocodiles and all sizes were represented. The habitat in San Blas estuaries allows Crocodylus acutus develop properly its biological cycle.
\end{abstract}

Key words: distribution, abundance, american crocodile, river crocodile.

\section{Introducción}

Se han realizado estudios de ecología y estatus de las poblaciones de Crocodylus acutus en todo su intervalo de distribución. Kushlan y Mazzotii (1989) estudian el uso de hábitat, dispersión de organismos, estructura y densidad de población en el sur de Florida; Mazzotii (1999) determina el esfuerzo de anidación así como el crecimiento y supervivencia de crías y juveniles de cocodrilos en la bahía de Florida; Richards et al. (2003) analizan mediante un modelo matemático la dinámica de la pobla-

Recibido: 13 agosto 2010; aceptado: 23 enero 2011 ción de cocodrilos en Florida; Thorbjarnarson (1989) realiza estudios de distribución y abundancia en 3 poblaciones, Florida, Haití y Republica Dominicana; Seijas (1990), la distribución, densidad, estatus poblacional y medidas de conservación en Venezuela; Rodríguez-Soberón (2000), la distribución, tamaño de las poblaciones y zonas de anidación en Cuba; Buitrago (2003), la distribución, densidad y tendencia de población así como aprovechamiento de la especie y lineamientos de conservación en Nicaragua, y Thorbjarnason et al. (2006) estimaron el tamaño de las poblaciones en 9 bioregiones con una propuesta de 69 unidades de conservación de cocodrilos (siglas en inglés CCUs) a lo largo del intervalo de distribución de la especie. 
En México, Casas-Andreu y Guzmán-Arroyo (1970) y Álvarez del Toro (1974) mencionan la situación de $C$. acutus en todo el país. En estudios posteriores, diferentes autores, entre otros Casas-Andreu et al. (1990), informan sobre la situación de la especie, estiman la distribución y el tamaño de población, y describen las zonas de anidación en Jalisco. Cupul et al. (2002) informan sobre el tamaño y densidad poblacional en un estero de Puerto Vallarta, Jalisco; Martínez-Ibarra et al. (1997) estiman la densidad, uso de hábitat y zonas de anidación en la Reserva de la Biosfera de La Encrucijada en Chiapas; Sigler (1997) revisa la distribución, abundancia, anidación y uso de hábitat en 7 regiones fisiográficas de Chiapas; García-Grajales (2005) y Brandon-Pliego (2006) examina el tamaño de población, densidad, supervivencia, anidación y preferencia de hábitat en 2 cuerpos de agua en Oaxaca, y Charruau et al. (2005) determinan tamaño de población, densidad y uso de hábitat en la Reserva de la Biosfera Banco Chinchorro en Quintana Roo.

En el estado de Nayarit existen trabajos sobre ecología y conservación de cocodrilos como el de Romero-Villaruel (2002) en la presa de Aguamilpa, donde se describe como la especie se adaptó a un área modificada en un río. Para el caso de San Blas, desde la creación del Centro de Investigación de la Vida Silvestre, Cocodrilario La Palma, en 1985, se han desarrollado trabajos sobre reproducción, etología, colecta de nidos, reintroducción y liberación de cocodrilos juveniles en los diferentes humedales en la zona, así como atención en casos de "cocodrilos problema" en ataques a humanos o sus intereses (SEMARNAT, 2001). Lo anterior muestra que el estudio científico de las poblaciones animales se circunscribe a la rama de la ecología de poblaciones que investiga los procesos que regulan la distribución y abundancia de las especies (Krebs, 1985); esta información permite diseñar estrategias para su conservación y aprovechamiento.

El objetivo del presente estudio es caracterizar la población de cocodrilos a partir de su distribución y abundancia en los esteros de San Blas, Nayarit, lo que contribuirá al conocimiento de la especie y permitirá establecer los lineamientos y directrices de conservación.

\section{Materiales y métodos}

Área de estudio. Los esteros del municipio de San Blas se encuentran localizados al sur de la desembocadura del río Santiago y hasta el puerto de San Blas. El polígono está situado entre las coordenadas que van de $21^{\circ} 31^{\prime} 43.8^{\prime \prime}$ a $21^{\circ} 37^{\prime} 38.35^{\prime \prime} \mathrm{N}$ y de $105^{\circ} 12^{\prime} 22.61^{\prime \prime}$ a $105^{\circ} 24^{\prime} 5.63^{\prime \prime}$ O (Fig. 1). Estos humedales muestran las características clásicas de definición de lagunas costeras o esteros, donde se da la mezcla entre 2 masas de agua, una marina y otra continental (Contreras, 1993). Se trata de un cuerpo de agua semicerrado que tiene una conexión libre con el mar abierto y en el cual se diluye el agua marina con el agua dulce proveniente del valle formado por el río Santiago. En ambos sistemas se encuentran canales bien definidos, con varios metros de profundidad y lagunas someras, como laguna Pericos en el estero El Rey, albufera Singayta en el estero San Cristóbal y laguna El Carrizal en La Tobara. La vegetación predominante es manglar (Rhizophora mangle, Laguncularia racemosa, Avicenia germinans y Conocarpus erectus) con manchones de bosque de galería; en las zonas cercanas a los cerros existen mezclas de bosque tropical subcaducifolio, bosque tropical caducifolio y cultivos (plátano, mango y coco de agua). Estos sistemas tienen características propias para que exista una gran diversidad biológica (Flores-Verdugo et al., 1992; CONABIO, 1998 y CONANP, 2008).

Métodos. Se analizó la cartografía del Instituto Nacional de Estadística, Geografía e Informática (INEGI; mapas 1:250 000, 1:50 000 y fotografías aéreas 1:20 000) e imágenes de satélite Land Sat 2000, en las que se identificaron los esteros, canales y lagunas como hábitat disponible del cocodrilo. La vegetación se describió de acuerdo con lo propuesto por Rzedowski (1978).

En el presente estudio se trabajaron 2 sistemas: 1), San Cristóbal-La Tobara con una superficie de 5732.54 ha, en el que se realizaron 3 transectos: A (La Tobara) de $12 \mathrm{~km}$; B (San Cristóbal-Singayta) de 15 km, y C (Los NegrosZoquipan) de $15 \mathrm{~km}$, y 2), el sistema El Rey-Pozo-laguna Pericos con una superficie de 10397.76 ha, en el que se realizaron 3 transectos: D (El Pozo) de $15 \mathrm{~km}, \mathrm{E}$ (Pericos) de 14 km y F (El Rey) de 18 km.

Los recorridos se realizaron en junio de 2005 y febrero de 2006 (estiaje), y en octubre 2005 y octubre 2007 (lluvias). En total se efectuaron 6 recorridos diurnos por cada transecto, con un esfuerzo de muestreo de $96 \mathrm{~h}$ para un

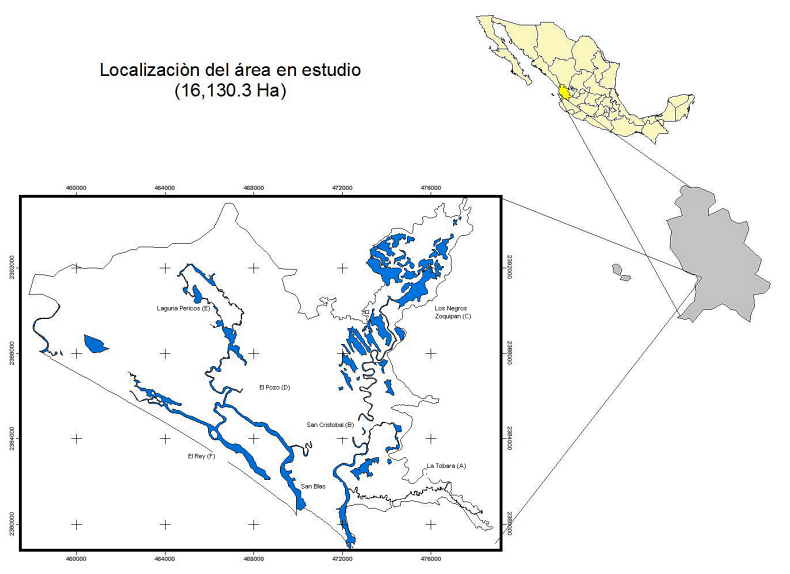

Figura 1. Área de estudio. 
total de $360 \mathrm{~km}$, donde se registraron zonas con nidos, cuevas, rastros y vegetación.

Los recorridos nocturnos se efectuaron durante 6 noches con un esfuerzo de muestreo de $168 \mathrm{~h}$ para un total de 356 kilómetros. En cada trayecto se contabilizaron los ejemplares observados para calcular su densidad relativa (ind $/ \mathrm{km}$ ). Debido al ancho y profundidad de los canales, se navegaron en una lancha de 14 pies de eslora con un motor fuera de borda de $25 \mathrm{hp}$ a una velocidad de $10 \mathrm{~km} / \mathrm{hr}$. Para el avistamiento, el observador iba en la proa de la lancha con lámpara de mano o frontal, de 6 voltios, iluminando el espejo de agua, los bordes del canal, bajo el manglar y encima de la vegetación emergente. En canales mayores a $50 \mathrm{~m}$ de ancho o en lagunas se utilizó un reflector (spot line) de 2 millones de candelas. La temperatura se tomó con un termómetro electrónico con intervalos de -40 a $150^{\circ} \mathrm{C}$. Para la salinidad se utilizó un refractómetro de mano con intervalos de 0 a $100 \%$ y precisión $1 \%$.

Para estimar el tamaño poblacional se utilizó el modelo propuesto por Messel et al. (1981), modificado por Thorbjarnarson et al. (2000), que consiste en calcular el valor porcentual de la población observada $\mathrm{P}$, a partir de la cual se estima la población total $\mathrm{N}$. Con lo anterior se obtiene una medida indirecta de la densidad total mediante el recuento directo de cocodrilos observados.

La primera expresión matemática para estimar la fracción de la población observada es:

$$
\mathrm{P}=\frac{\mathrm{m}}{(2 \mathrm{~s}+\mathrm{m}) 1.05}
$$

donde P es el porcentaje de la población observada, m es la media del número de cocodrilos observados en el total de los muestreos, s es la desviación estándar del número de cocodrilos observados para el total de muestreos y el nivel de error es 1.05 .

La segunda expresión matemática para calcular el tamaño total de la población $(\mathrm{N})$, con un nivel de confianza de $95 \%$, aceptando la normalidad de los conteos, es:

$$
\mathrm{N}=\frac{\mathrm{m}}{\mathrm{p}} \pm \frac{[1.96(\mathrm{~s})] 1 / 2}{(2 \mathrm{~s}+\mathrm{m}) 1.05}
$$

donde 1.96 es el valor crítico tomado de $\mathrm{F}$ al $95 \%$ de confiabilidad.

La tasa promedio de encuentro (densidad relativa) de la población se calculó determinando el número de cocodrilos observados por kilómetro lineal (cocodrilos $/ \mathrm{km}$ ). La estructura poblacional se determinó al observar el reflejo de la luz de la lámpara en la lámina reflectora (tapetum lucidum) de los ojos de los cocodrilos. La separación de los ojos, así como la distancia de éstos a la punta del hocico permiten estimar visualmente la talla aproximada del animal (Messel et al., 1981; Thorbjarnarson, 1989). De acuerdo con
Sigler (1999) y Platt y Thorbjarnarson (2000) una vez que se observaron los cocodrilos y se estimó su longitud total, se clasificaron como clase I neo (LT $=<30 \mathrm{~cm}$, neonatos), clase I $(\mathrm{LT}=31-60 \mathrm{~cm})$, clase II $(\mathrm{LT}=61-120 \mathrm{~cm})$, clase III $(\mathrm{LT}=121-180 \mathrm{~cm})$, clase IV $(181-240 \mathrm{~cm})$ y clase V $(>240 \mathrm{~cm})$ y ojos (longitud no determinada).

Con la finalidad de determinar si la abundancia de los cocodrilos responde a características como hidrografía, profundidad y vegetación, así como temperatura y salinidad, por temporada muestreada y transecto, es decir, para determinar patrones de abundancia en la zona, se realizó un análisis de escalamiento multidimesional nométrico (MDS) utilizando el índice de Bray Curtis $(p=$ 0.05) (Clarke y Warwick, 2001 y Guerrero-Casas y Ramírez-Hurtado, 2002). El análisis genera un coeficiente de significancia; si el coeficiente es menor de 0.1 , indica que los grupos formados son significativamente diferentes en su composición, pero si es mayor de 0.1 , tienen la misma composición (no hay diferencias significativas, Clarke y Warwick, 2001). Para lo anterior se creó una base de datos y se empleó el software package Paleotological Statistics (PAST) en su versión 2.00 (1999-2010).

\section{Resultados}

La distribución de los cocodrilos en los transectos se presentó en grupos o agregados, siendo mayor en los transectos A, B y C, los cuales son canales con más de $1 \mathrm{~m}$ de profundidad durante todo el año; además, presentan meandros, lo que disminuye la corriente y facilita la formación de playas que sirven de asoleadero a los cocodrilos. Esta zona tiene una topografía más elevada cercana al canal, donde la vegetación se presenta en mosaicos de bosque de galería, bosque tropical subcaducifolio y cultivos. La vegetación acuática fue principalmente manglar (Rhizophora mangle, Laguncularia racemosa) y manchones de tular (Typha dominguesis). Este sistema presenta importantes aportes de agua dulce, la salinidad promedio en estiaje fue de $11.03 \%$ y en lluvias de $4.92 \%$, la temperatura promedio del agua fue de $26.72^{\circ} \mathrm{C}$ durante el estiaje y de $29.85^{\circ} \mathrm{C}$ en lluvias.

Los transectos D, E y F presentaron menor distribución de cocodrilos; son áreas que forman parte de la planicie costera con zonas inundables, la vegetación fue manglar (L. racemosa y A. germinans con algunos manchones de $R$. mangle). Estos transectos presentaron grandes afluencias de agua marina; la salinidad promedio registrada en estiaje fue de $35.85 \%$ y de $24.56 \%$ en lluvias; la temperatura promedio del agua en estiaje fue de $27.64^{\circ} \mathrm{C}$ y de $30.93^{\circ} \mathrm{C}$ en lluvias.

Dentro de las zonas de distribución se localizaron 26 nidos activos en los transectos $\mathrm{A}$ y $\mathrm{C}$ en 3 sitios dife- 
Cuadro 1. Características de los nidos en el sistema estuarino San Cristóbal-La Tobara-Los Negros

\begin{tabular}{ccccccc}
\hline Transecto & Hábitat & $\begin{array}{c}\text { Núm. } \\
\text { nidos }\end{array}$ & $\begin{array}{c}\text { Profundidad } \\
\text { promedio } \pm \text { DE) } \\
m\end{array}$ & $\begin{array}{c}\text { Diámetro } \\
\text { promedio } \pm \text { DE) } \\
m\end{array}$ & $\begin{array}{c}\text { Distancia al } \\
\text { agua }\end{array}$ & $\begin{array}{c}\text { Nivel sobre agua } \\
\text { (promedio e intervalo) } m\end{array}$ \\
\hline A & Carretera y & 12 & 0.47 & 0.41 & 0.96 \\
$($ pa Tobara) & construcción & & \pm 0.08 & \pm 0.06 & 2.6 & $0.4-1.5$ \\
A & Cerca o en & 6 & 0.4 & 0.34 & 30.5 & 4.9 \\
$($ La Tobara) & cultivos & & \pm 0.07 & \pm 0.05 & $2-150$ & $7-20$ \\
C & $\begin{array}{c}\text { Canales y } \\
\text { lagunas, }\end{array}$ & 8 & 0.48 & 0.45 & 8.83 & 7.83 \\
pocos cultivos & & \pm 0.07 & \pm 0.12 & $2-19.5$ & $0.4-1$ \\
\hline
\end{tabular}

DE, desviación estándar; m, metros.

rentes (Cuadro 1). El sustrato encontrado fue de arena, arena-grava, arena-roca, arcilla, grava, limo, limo-arenoso, limo-arcilloso y limo-roca. La vegetación fue bosque de galería, bosque tropical subcaducifolio, cultivo, manglar y pastos.

En el transecto $\mathrm{C}$ se identificó una zona con 20 cuevas denominada Los Negros. La distancia entre las cuevas más alejadas fue de $2.33 \mathrm{~km}$. La distancia promedio de las cuevas a la zona de nidos de la misma área fue de 10 $\mathrm{m}$. La vegetación que se registró fue bosque de galería, bosque tropical subcaducifolio y manglar. El sustrato fue limo-arcilloso.

La mayor abundancia se registró en los transectos A, B y $\mathrm{C}$ con $\mathrm{N}=311.62$ cocodrilos y una densidad relativa que osciló entre 2.68 y $4.31 \mathrm{ind} / \mathrm{km}$; sólo aquí se registraron neonatos. En D, E y F se estimó una $\mathrm{N}=21.79$ cocodrilos y una densidad relativa que osciló entre 0.014 y $0.36 \mathrm{ind} / \mathrm{km}$; en estos transectos no se observaron neonatos (Cuadro 2).

El análisis MDS sobre los patrones de abundancia y densidad de los cocodrilos en los 6 transectos nos dan una similitud $(\mathrm{p}=0.05)$ del $10 \%$ con un coeficiente de 0 , representando que los grupos formados tienen diferencias significativas. El análisis MDS por dendograma arrojó la formación de los siguientes 3 grupos, donde la similitud fue para $\mathrm{A}, \mathrm{B}$ y $\mathrm{C}=80 \%, \mathrm{D}$ y $\mathrm{E}=70 \%$ y $\mathrm{F}=10 \%$ respecto a los otros grupos (Figs. 2 y 3 ).
La estructura de la población por transecto se registró en el Cuadro 3; en A, B y $\mathrm{C}$ se encontraron los recién eclosionados ( $\mathrm{I}$ neo) con una $\mathrm{N}=152.77$ cocodrilos, la suma de crías (clases I neo y I) registró una $\mathrm{N}=200.7$; en la clase III se estimó una $\mathrm{N}=16.44$, menor a las clases II con una $\mathrm{N}=49.98$ y IV con una $\mathrm{N}=59.67$, y en las clases reproductoras IV y $\mathrm{V}$ se estimó una $\mathrm{N}=104.34$. En los cocodrilos que no se logró estimar la talla (ojos) se registró una $\mathrm{N}=44.04$.

En los transectos D, E y F, no se registró la clase I neo, en la clase I se estimó una $\mathrm{N}=1.74$, en las clases II y III se estimó una $\mathrm{N}=18.63$ y en las clases IV y V se estimó $\mathrm{N}=$ 5.42. En los cocodrilos que no se logró estimar la talla se registraron con $\mathrm{N}=2.62$. (Figs. 4 y 5 ).

\section{Discusión}

El comportamiento de la distribución y abundancia está en función de las características biológico-ambientales, como alimentación, reproducción, temperatura y salinidad (Kushlan y Mazzotti, 1989; Thorbjarnarson, 1989). Aunque el objetivo del presente estudio no fue evaluar los hábitos alimenticios de los cocodrilos, durante los recorridos en el área de estudio se observaron peces, reptiles, aves y mamíferos medianos (Procyon lotor y Nasua narica) que forman parte de la dieta de los cocodrilos. Incluso a las

Cuadro 2. Abundancia y tasa promedio de encuentro de la población de cocodrilos

\begin{tabular}{ccccccc}
\hline & \multicolumn{3}{c}{ Sistema San Cristóbal-La Tobara } & \multicolumn{3}{c}{ Sistema Rey-Pozo-laguna Pericos } \\
Transecto & $A$ & $B$ & $C$ & $D$ & $E$ & $F$ \\
\hline $\mathrm{Km}$ & 12 & 15 & 15 & 15 & 14 \\
Organismos observados $\sum$ 4 recorridos & 207 & 161 & 250 & 22 & 10 & 1 \\
Tasa promedio de encuentro (ind/km) & 4.31 & 2.68 & 4.16 & 0.36 & 0.17 & 0.014 \\
Porcentaje observado (P) & 54 & 59 & 42 & 44 & 31 \\
Tamaño de población (N) & $95.5 \pm 8.42$ & $68.1 \pm 6.39$ & $148.02 \pm 13.5$ & $12.3 \pm 3.69$ & $8.18 \pm 4.11$ & $1.31 \pm 2.27$ \\
\hline
\end{tabular}


Cuadro 3. Estructura de la población de cocodrilos con los datos de N (tamaño de población) por transecto

\begin{tabular}{cccccccc}
\hline & & & \multicolumn{2}{c}{ Clases } & & ojos \\
Transecto & Ineo & $I$ & $I I$ & $I I I$ & $I V$ & V \\
\hline A & 30.46 & 10.15 & 23.45 & 5.0 & 21.93 & 19.39 & 13.31 \\
B & 36.36 & 7.16 & 13.43 & 6.11 & 14.84 & 9.97 & 17.8 \\
C & 85.95 & 30.63 & 13.1 & 5.34 & 22.9 & 15.31 & 12.93 \\
D & 0 & 1.74 & 8.67 & 3.84 & 1.31 & 1.31 & 0 \\
E & 0 & 0 & 3.5 & 2.62 & 2.8 & 0 & 1.31 \\
F & 0 & 0 & 0 & 0 & 0 & 0 & 1.31 \\
\hline
\end{tabular}

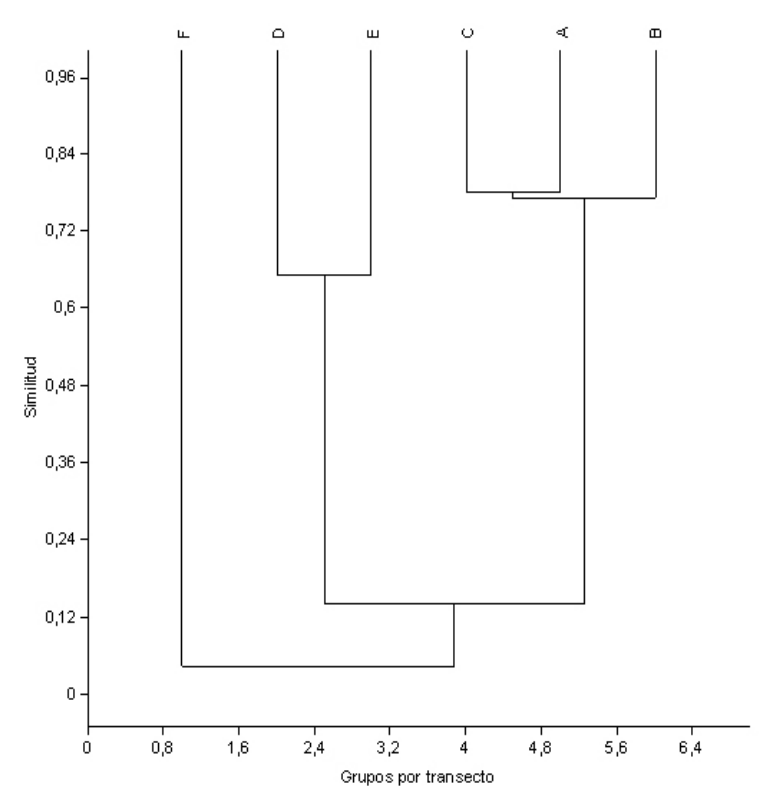

Figura 2. Análisis MDS por dendograma para abundancia de cocodrilos en los 6 transectos: A, La Tobara; B, San Cristóbal; C, Los Negros-Zoquipan; D, El Pozo; E, canal y laguna Pericos; F. El Rey.

crías se les observó cazando pequeños cangrejos y peces. Por lo que se tomó el parámetro de la alimentación como uniforme en toda el área de estudio.

La mayor distribución de los cocodrilos en los transectos $\mathrm{A}, \mathrm{B}$ y $\mathrm{C}$ con una $\mathrm{N}=311.62$ cocodrilos se registró de acuerdo con las características ambiéntales, como agua permanente y profundidad $>1 \mathrm{~m}$, corriente lenta, baja salinidad entre 4.92 y $11.03 \%$, topografía más elevada con un mosaico de 6 tipos de vegetación. En contraparte, los transectos D, E y F con una $\mathrm{N}=21.79$ cocodrilos, presentaron canales más anchos con mayor flujo de agua y alta salinidad, entre 24.76 y $35.85 \%$, con topografía de planicie costera y un solo tipo de vegetación. Las características ambientales anteriores coinciden con los estudios de Kushlan y Mazzotti (1989) en el sur de Florida, donde los cocodrilos se distribuyen en pantanos, arroyos, bahías

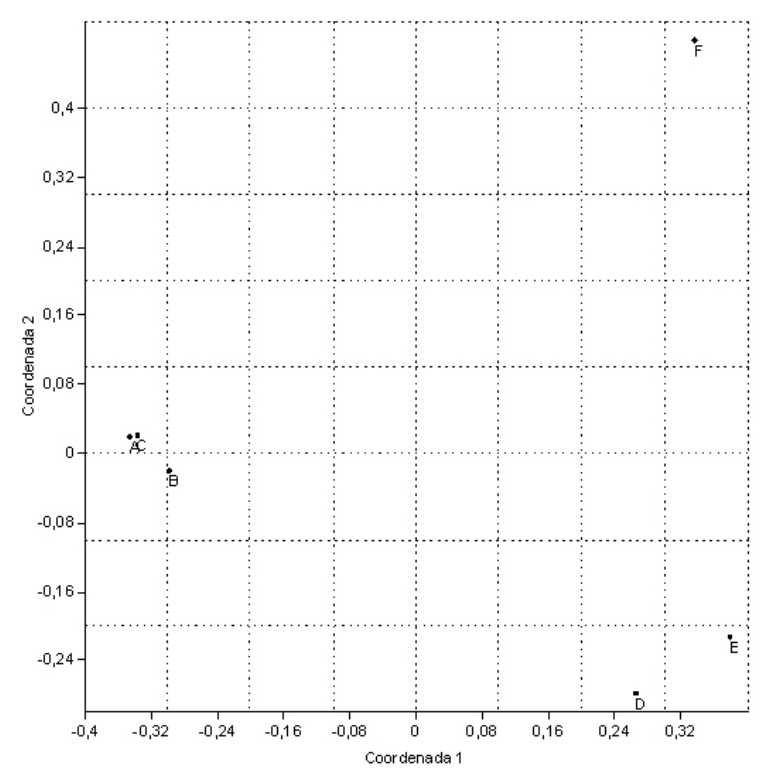

Figura 3. Análisis MDS por conglomerados para abundancia de cocodrilos en los 6 transectos, coeficiente de estrés=0: A, La Tobara; B, San Cristóbal; C, Los Negros-Zoquipan; D, El Pozo; E, canal y laguna Pericos; F, El Rey.

y en aguas protegidas de la línea costera con salinidades de $14 \%$.

La salinidad es un parámetro importante en la distribución de la especie. En Florida, Dunson (1982) experimentó con crías de C. acutus a diferentes salinidades, obteniendo que no toleran salinidades por encima de 35\%, y en el sur de Florida, Dunson y Mazzotii (1989) observaron adultos de C. acutus en salinidades que oscilaron enre 10 y $18 \%$. Hernández-Hurtado et al. (2006) observaron cocodrilos en salinidades de $41 \%$ en los esteros de bahía de Banderas entre Jalisco y Nayarit, y Thorbjarnarson (1989) los vio en el lago Enriquillo de República Dominicana a salinidades de 78\%.

Richards et al. (2004), en el sur de Florida, realizaron un modelo de salinidad para C. acutus relacionado con la distribución y abundancia de cocodrilos en una proyec- 
ción a 500 años. Encontraron que la salinidad en la que habría un mayor número de cocodrilos sería de 0 a $20 \%$. Con los datos anteriores se observó que la población de cocodrilos en San Blas tiene una preferencia de salinidad similar a la de los cocodrilos del sur de Florida.

Las características de los nidos del presente estudio, que se mencionan en el Cuadro 1, están dentro de lo registrado para C. acutus por Thorbjarnarson (1989), Casas-Andreu (2003), Cupul et al. (2004) y HernándezHurtado et al. (2006): diámetro de la cama entre 0.89 y 2 $\mathrm{m}$, con profundidades de 17 a $68 \mathrm{~cm}$, diámetro del nido de 25 a $76 \mathrm{~cm}$, distancia al agua de 0.5 a $150 \mathrm{~m}$, nivel sobre el agua de 0.4 a $20 \mathrm{~m}$. Los sustratos registrados fueron arenoso, limo-arcilloso, grava, con vegetación, suelos pedregosos y material de relleno para caminos y carreteras.

Para el sur de Florida, en un área de 12000 ha, Mazzotti (1999) registró 101 nidos anualmente. CasasAndreu (2003) en un área de 1000 ha en Cuitzmala, Jalisco, registró 39 nidos por año. En el presente estudio se registraron 26 nidos activos anuales en un área de 5 732.54 ha dentro de los transectos A, B y C, por lo que se considera que existe hábitat potencial para la anidación. Los nidos al borde de carreteras se encuentran en riesgo para los recién eclosionados, lo que requiere de investigación a largo plazo.

Las cuevas se localizaron en el margen este del transecto $\mathrm{C}$, el cual es más elevado, formándose un bordo que facilita que los cocodrilos caven. Álvarez del Toro (1974) y Thorbjarnarson (1989) mencionan que los cocodrilos utilizan las cuevas como sitios de refugio, descanso y termorregulación en estiajes prolongados. Además la cercanía de las cuevas con la zona de anidación de Los Negros permite que funcionen como sitios centinela, para que las hembras lleven a cabo los cuidados parentales y de protección a los nidos.

La densidad relativa en los transectos A, B y C fue estadísticamente similar $(80 \%)$, lo anterior se ve respaldado por observaciones en la ecología reproductiva, 2 zonas de anidación con su grupo reproductivo cada una y presencia de neonatos. Lo anterior permite definir este grupo como una población de cocodrilos. Además, las características ambientales, como la vegetación heterogénea con topografía elevada y baja salinidad, permiten definir estos transectos como sistema San Cristobal-La Tobara.

En contraparte, se observó que la densidad relativa en los transectos D, E y F y la de los transectos A, B y C son estadísticamente disímiles (90\%).

Los transectos D y E son similares (70\%), y F, donde sólo se registró un cocodrilo durante el estudio, es disímil $(90 \%)$ a los demás transectos. Lo anterior, aunado a la poca abundancia de cocodrilos (21.79 individuos), exclu- sión de neonatos y zonas reproductivas, permite definirlos como un subgrupo de la población estimada. Las características ambientales, como la vegetación homogénea con planicie costera y alta salinidad, permitió definir los transectos D, E y F como el sistema El Rey-Pozo-Pericos.

Las tasas promedio de encuentro en toda el área de estudio oscilaron entre 0.014 y $4.31 \mathrm{ind} / \mathrm{km}$, donde la estimación de la población en conjunto fue de 333 individuos en un área de 16130.3 ha, resultados semejantes a los de estudios realizados en hábitats costeros, como el de Martínez-Ibarra et al. (1997) en la Encrucijada (11.5 km) que registra $2.1 \mathrm{ind} / \mathrm{km}$ y difiere de lo registrado por Valtierra (2001) para Cuitzmala (10.68) con 14 ind $/ \mathrm{km}$. Cabe mencionar que estos estudios se realizaron en áreas naturales protegidas, lo que puede favorecer las poblaciones de cocodrilos.

Las densidades por área para los transectos $\mathrm{A}, \mathrm{B}$ y $\mathrm{C}$ fue de $0.05 \mathrm{ind} /$ ha y para los transectos D, E y F fue de $0.002 \mathrm{ind} / \mathrm{ha}$. La densidad del primer grupo de transectos es similar a las reservas naturales registradas por Rodríguez-Soberón (2000) en el golfo de Guacanayabo en Cuba con 0.02 ind/ha y Kushlan y Mazzotti (1989) en Florida con 0.025 ind/ha. Cabe destacar que estas poblaciones con 7500 y 1000 individuos respectivamente, están consideradas como las mejor conservadas en todo el intervalo de distribución de C. acutus.

En la estructura de la población de cocodrilos en los transectos A, B y C están bien representadas todas las clases salvo la III, en la cual se estimó una abundancia baja $(\mathrm{N}=16.34$ cocodrilos). Lo anterior se interpretó como una población bien establecida en las clases reproductoras, con buen aporte de crías y juveniles, similar a lo que registran Álvarez del Toro (1974), Kushlan y Mazzotti (1989), Thorbjarnarson (1989) y Richards et al. (2003), donde mencionan que los organismos con tallas que oscilan entre 100 y $200 \mathrm{~cm}$ son la fase biológica que más dispersión presenta en una población, refugiándose en manglares y sitios intrincados de difícil acceso para el humano; las grandes pérdidas de cocodrilos en clases I y II explican por qué existen menos cocodrilos en la III. La clase I neo aportó 153 crías al año, que se interpretó como un aporte de 6 crías por nido. La ausencia de neonatos (I neo) en los transectos D, E y F, y la mayor abundancia en las clases II y III reforzó la tesis de que los cocodrilos de estos transectos son un subgrupo de la población de los transectos A, B y C (Figs. 4 y 5).

El presente estudio contribuye al conocimiento de la ecología poblacional de C. acutus en los esteros de San Blas, Nayarit, donde el tamaño de población se estimó en 333 cocodrilos y la densidad osciló entre 0.014 y 4.31 ind $/ \mathrm{km}$ o 0.002 y $0.05 \mathrm{ind} / \mathrm{ha}$, el hábitat disponible permite realizar su ciclo biológico. La mayor distribución de 


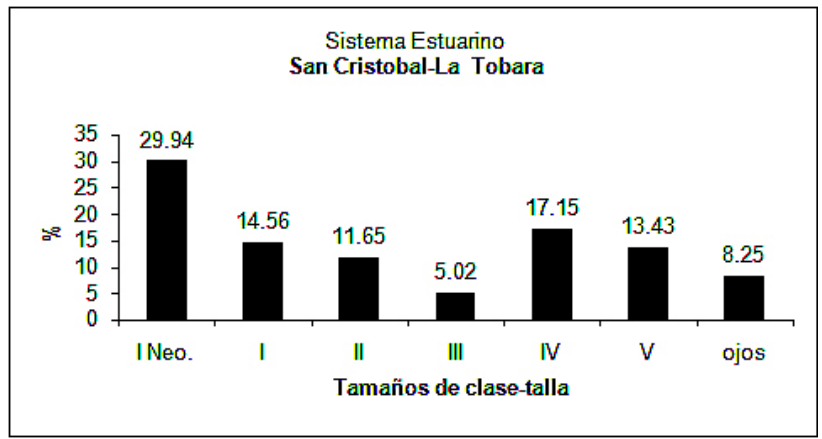

Figura 4. Estructura poblacional por clases de tamaño (Longitud total) para el sistema "San Cristóbal- La Tobara".

cocodrilos se caracterizó por presentarse en canales con profundidad $>1 \mathrm{~m}$, vegetación heterogénea, topografía elevada y baja salinidad. Sin embargo, se debe continuar con los esfuerzos de investigación, planteando un seguimiento del estudio a largo plazo.

\section{Agradecimientos}

A las siguientes personas, por su apoyo y ayuda en el desarrollo de esta investigación: maestro Juan Luis Cifuentes Lemus, Dr. Irán Bojórquez, Dra. Elaine Espino, Dr. Clemente Lemus, MVZ. Carlos Villar, M.C. Oyolsi Nájera, M.C. Fernando Flores, Ocean. Isabel Cárdenas, Ocean. Rafael García de Quevedo, Dr. Amilcar Cupul y Dr. Fabio Cupul. También al Biól. Jerónimo Domínguez e Ing. Raymundo Ramírez.

\section{Literatura citada}

Álvarez del Toro, M. 1974. Los Crocodylia de México. Instituto Mexicano de Recursos Naturales Renovables, Consejo Nacional de Ciencia y Tecnología y World Wildlife Fund, México, D.F. 70 p.

Brandon-Pliego, V. R. 2006. Tamaño poblacional del Crocodylus acutus (Cuvier, 1807) (Reptilia: Crocodylia) y su variación interanual en la laguna La Palmita, Jamiltepec, Oaxaca, durante el periodo 2004-2005. Tesis, Universidad del Mar, Campus Puerto Ángel. 52 p.

Buitrago, F. 2003. Aprovechamiento de los cocodrilos de Nicaragua. Cuadernos de Investigación 11, Universidad Centroamericana de Managua, Nicaragua. 75 p.

Casas-Andreu, G. y M. Guzmán-Arroyo. 1970. Estado actual de las investigaciones sobre cocodrilos mexicanos. Instituto Nacional de Investigación Biológico Pesqueras, Secretaría de Industria y Comercio, México, D. F. p. 1-50.

Casas-Andreu, A. G., T. Reyna-Trujillo y F. R. Méndez de la Cruz. 1990. Estado actual de Crocodylus acutus en la costa del Pacífico de México. Revista de la Sociedad Mexicana

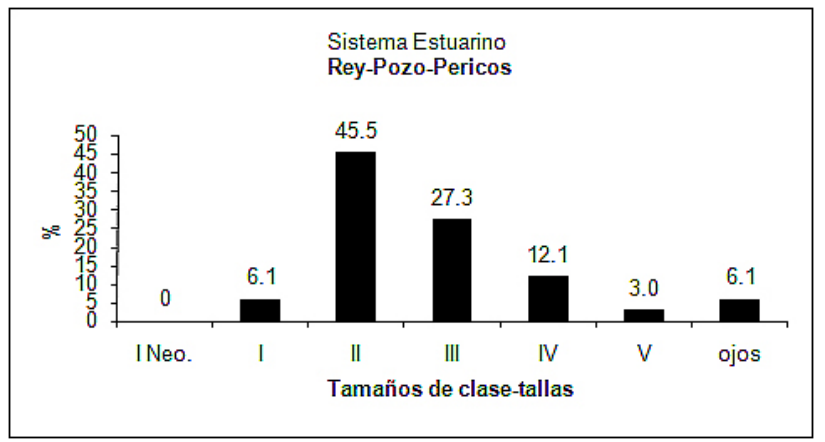

Figura 5. Estructura poblacional por clases de tamaño (Longitud total) para el sistema "Rey-Pozo-Laguna Pericos".

de Historia Natural 41:57-62.

Casas-Andreu, G. 2003. Ecología de anidación de Crocodylus acutus (Reptilia: Crocodylidae) en la desembocadura del río Cuitzmala, Jalisco, México. Acta Zoológica Mexicana (Nueva Serie) 89:111-127.

Charruau, P., J. R. Cedeño-Vázquez y S. Calme. 2005. Status and Conservation of the American Crocodile (Crocodylus acutus) in Banco Chinchorro Biosphere Reserve, Quintana Roo, México. Herpetological Review 36:390-395.

Clarke, K. R. y R. M. Warwick. 2001. Change in Marine Comunities: An approach to statistical analysis and interpretation. Primer-E, Plymouth. 172 p.

CONABIO (Comisión Nacional para el Conocimiento y Uso de la Biodiversidad). 1998. Regiones prioritarias marinas de México. CONABIO, México. D. F. 81 p.

CONANP (Comisión Nacional de Áreas Naturales Protegidas). 2008. Estudio previo justificativo para el establecimiento del Área Natural Protegida, Área de Protección de Flora y Fauna "Sistema Singayta, La Tovara y Los Negros", San Blas, Nayarit. 186 p.

Contreras, F. 1993. Ecosistemas costeros mexicanos. Comisión Nacional para el Conocimiento y Uso de la Biodiversidad/ Universidad Autónoma Metropolitana-Unidad Iztapalapa, México, D.F. 415 p.

Cupul, F., A. Rubio., A. Reyes y H. Hernández. 2002. Sondeo poblacional de Crocodylus acutus (Cuvier, 1807) en el estero Boca Negra, Jalisco. Universidad del Mar, Revista Ciencia y Mar 6:45-50.

Cupul, F., A. De Niz. A. Reyes y A. Rubio. 2004. Historia natural del cocodrilo americano (Crocodylus acutus) en el estero Boca Negra, Jalisco, México: anidación y crecimiento de neonatos. Universidad del Mar, Revista Ciencia y Mar 8:31-42.

Dunson, W. A. 1982. Salinity relations of crocodiles in Florida Bay. Copeia 374-385.

Dunson, W. A. y F. J. Mazzotti. 1989. Salinity as a limiting factor in the distribution of reptiles in Florida Bay: a theory for the estuarine origin of marine snakes and 
turtles. Bulletin of Marine Science 44:229-244.

Flores- Verdugo, F., F. González-Farías, D. Segura-Zamorano y P. Ramírez-García. 1992. Mangrove ecosystems of the Pacific coast of México: distribution, structure, litterfall and detritus dynamics. In Coastal plant communities of Latin America, Seelger (ed.). Academic, New York. p. 269-288.

García-Grajales, J. 2005. Diseño de una estrategia de manejo del cocodrilo americano (Crocodylus acutus) en el estero La Ventanilla, Oaxaca, México. Tesis, Maestría Instituto de Ecología, Xalapa, Veracruz. 96 p.

Guerrero-Casas, F. M y J. M. Ramírez-Hurtado. 2002. El análisis de escalamiento multidimensional: una alternativa y un complemento a otras técnicas multivariantes. Memorias de X Jornadas de la Asociación Española de Profesores Universitarios de Matemáticas para la Economía y Empresa. http://www.uv.es/asepuma/X/ comunica.htm. ultima consulta 23.IV.10.

Hernández-Hurtado, H., R. García de Quevedo M. y P. Hernández-Hurtado. 2006. Los cocodrilos de la costa Pacífico occidental (Michoacán, Colima y Jalisco) de México. In Los recursos pesqueros y acuícolas de Jalisco, Colima y Michoacán, M. C. Jiménez-Quiroz y E. Espino-Bar (eds.). Secretaría de Agricultura, Ganadería, Desarrollo Rural, Pesca y Alimentación/ Instituto Nacional de la Pesca/ Centro Regional de Investigaciones Pesqueras-Manzanillo, Colima. p. 375-388.

Krebs, C. J. 1985. Ecología, estudio de la distribución y abundancia. Harla, México, D. F. 753 p.

Kushlan, J. A. y F. J. Mazzotti. 1989. Population biology of the American crocodile. Journal of Herpetology 23:7-21.

Martínez-Ibarra, J. A., E. Naranjo y C. Nelson. 1997. Las poblaciones de cocodrilos (Crocodylus acutus) y caimanes (Caiman crocodilus) en una zona pesquera de la reserva de biosfera "La Encrucijada", Chiapas, México. Vida Silvestre Neotropical 6:21-28.

Mazzotti, F. J. 1999. The American Crocodile in Florida Bay. Estuaries 22:552-561.

Messel, H., J. C. Vorlicek, A. G. Wells and W. J. Green. 1981. Surveys of tidal river systems in the northern territory of Australia and their crocodile populations. Monograph 1. Pergamon, Sydney. 463 p.

Platt, S. G. y J. Thorbjarnarson. 2000. Status and conservation of the American crocodile, Crocodylus acutus, in Belice. Biological Conservation 96:13-20.

Richards, P. M., W. M. Mooij y D. L. De Angelis. 2003. Evaluating the relative effects of life history stages in the conservation of the American Crocodile (Crocodylus acutus) in Florida. Florida Scientist 66:273-286.

Richards, P. M., W. M. Mooij y D. L. De Angelis. 2004. Evaluating the effect of salinity on a simulated American Crocodile (Crocodylus acutus) population with applications to conservation and Everglades restoration. Ecological Modelling 180:371-394.

Rodríguez-Soberón, R. 2000. Situación actual de Crocodylus acutus en Cuba. Proceedings of the 15th Working Meeting of the Crocodile Specialist Group, International Union for Conservation of Nature - The World Conservation Union, Gland and Cambridge. p. xvii, 17-32.

Romero-Vllarruel. J., I. I. Martínez B., A. Razo P. y J. A. Cervantes R. 2002. Estudio de la población y distribución del cocodrilo de río (Crocodylus acutus) en el embalse de la presa de Agua Milpa, Nayarit. Memorias de la Cuarta Reunión del Subcomité del Proyecto de Conservación, Manejo y Aprovechamiento Sustentable de los Crocodylia de México (COMACROM). Universidad de Autónoma de Campeche y Centro de Estudios Tecnológicos del MarCampeche, p. 17-28.

Rzedowski, J. 1978. Vegetación de México. Limusa, México, D. F. 432 p.

Seijas, A. E. 1990. Status of the American crocodile in Venezuela. Proceedings of the 9th Working Meeting of the Crocodile Specialist Group, International Union for Conservation of Nature - The World Conservation Union, Gland and Cambridge. p iv, 144-156.

SEMARNAT (Secretaría del Medio Ambiente y Recursos Naturales). 2001. Programa operativo del Centro Reproductor de Cocodrilos La Palma. Secretaría de Medio Ambiente y Recursos Naturales, Delegación Nayarit, Tepic, Nayarit. 53 p.

Sigler, L. 1997. Poblaciones silvestres de cocodrilianos en el estado de Chiapas. Memorias de la Cuarta Reunión Regional del Grupo de Especialistas en Cocodrilos de América Latina y el Caribe, Villa Hermosa, Tabasco. p.189-192 .

Sigler, L. 1999. Conservación del cocodrilo de río Crocodylus acutus en el Parque Nacional "Cañón del Sumidero", Chiapas. Instituto de Historia Natural/ Gobierno del Estado de Chiapas/ Fondo Mexicano para la Conservación de la Naturaleza, Tuxtla Gutiérrez. 44 p.

Thorbjarnarson, J. 1989, Ecology of the American crocodile, Crocodylus acutus. In Crocodiles, their ecology, management and conservation.IUCN Publications New Series, Gland. p. 228-259.

Thorbjarnarson, J., S. Platt y U. Saw Tun Khaing. 2000. A population survey of the estuarine crocodile in the Ayeyarwady Delta, Myanmar. Orix 34:317-324.

Thorbjarnarson, J., F. Mazzotti, E. Sanderson, F. Buitrago, M. Lazcano, K. Minkowski, M. Muñiz, P. Ponce, L. Sigler, R. Soberón, A. M. Trelancia y A. Velasco. 2006. Regional habitat conservation priorities for the American crocodile. Biological Conservation 128:25-36.

Valtierra, A. M. 2001. Estado actual de la población de Crocodylus acutus en la Reserva de Biosfera Chamela- 
Cuixmala; trece años de protección: recomendaciones para un manejo sostenible. Memorias de la Tercera Reunión del Subcomité del Proyecto de Conservación, Manejo y Aprovechamiento Sustentable de los Crocodylia de México (COMACROM), Secretaría de Medio Ambiente y Recursos Naturales, Culiacán, Sinaloa. p. 61-68. 